\title{
Solar Array Technologies for Planetary Science and Astrobiology Missions
}

\author{
Primary author: Joel A. Schwartz \\ Phone \# 1-626-840-9037 \\ E-mail: joel.a.schwartz@jpl.nasa.gov \\ Jet Propulsion Laboratory, California Institute of Technology \\ Coauthors: $\quad$ Richard Ewell (Jet Propulsion Laboratory, California Institute \\ of Technology) \\ Nancy Haegel (National Renewable Energy Laboratory) \\ Simon Liu (The Aerospace Corporation) \\ Jeremiah Mcnatt (NASA Glenn Research Center) \\ Edward Plichta (General Technical Services, LLC) \\ Subbarao Surampudi (Jet Propulsion Laboratory, California \\ Institute of Technology)
}

The information in this white paper about future NASA mission concepts and their power system options is pre-decisional and is provided for planning and discussion purposes only.

A portion of this research was carried out at the Jet Propulsion Laboratory, California Institute of Technology, under a contract with the National Aeronautics and Space Administration (80NM0018D0004). 


\section{Solar Array Technologies for Planetary Science and Astrobiology Missions}

\section{Introduction}

Future planetary science and astrobiology missions will depend on advanced power generation technologies to enable long mission durations and survival in unique, challenging environments throughout the solar system ${ }^{1}$. In several cases, the ability to generate power in these environments will enable new missions that were previously considered unattainable. Advancing solar array technology is expected to play a significant role in these developments. The purpose of this paper is to evaluate how well current solar array technology can meet the needs of future missions and identify advances in solar array technology that can enable or enhance many mission concepts.

Planetary science and astrobiology missions frequently share several key needs in common with commercial and defense space applications. Both can require high efficiency solar cells and lightweight arrays to maximize the capability of payloads. Large, high power arrays would also be needed as mission payloads grow. For missions to the outer planets, larger arrays could be needed to provide sufficient power generation far from the sun where the solar irradiance is low. Large arrays would also enable solar electric propulsion (SEP) for many missions. Lower cost solar cells and arrays are also critical, particularly as array size and, therefore, cost increases.

In addition to shared technology needs, many planetary science and astrobiology missions have unique needs, resulting from the unique environments in which these missions operate. These include low irradiance, low temperature (LILT) conditions which limit available energy and also impact solar cell efficiency at distances far from the sun. At Saturn ( 10 AU), solar irradiance is $\sim 1 \%$ of that in Earth orbit. Extreme temperatures, reaching $\sim 33$ deg $\mathrm{K}$ during eclipse at the outer planets, also present unique challenges. At the inner planets and close to the sun, high irradiance results in high temperatures and unique thermal control challenges. In the lower Venus atmosphere, and on the Venus surface, a unique combination of low irradiance, high temperature (LIHT) conditions and corrosive gases at high pressure present a significant challenge for solar array technology. On the surface of Venus, atmospheric temperature is 465 deg C, pressure is 93 bar and solar irradiance is $\sim 6.5 \%$ of that in Earth orbit. On the lunar and Martian surfaces, dust accumulation presents a unique challenge for long-term, reliable operation.

Planetary radiation belts present another challenge for solar arrays as they gradually degrade the solar array power generation capability. For example, Jupiter's radiation belts are much more intense than Earth's Van Allen Belts. Other planets, such as Saturn, have planetary radiation belts comparable to Earth. Radiation hard solar cells and arrays would enable, enhance, and prolong missions in high radiation environments.

\section{State-of-Practice Space Solar Arrays}

State-of-practice (SoP) solar cells for Earth-orbiting missions include solar cell efficiencies from $29 \%$ to $32 \%$ at air mass zero (AM0, i.e. above the Earth's atmosphere), beginning-of-life, 28 deg C. These are multi-junction cells fabricated using III-V materials (e.g. GaInP 2 and GaInAs). The highest efficiency SoP cells use an inverted metamorphic (IMM) structure ${ }^{2}$. SoP arrays vary in specific power from $\sim 80 \mathrm{~W} / \mathrm{kg}$ for rigid panel arrays to $\sim 150 \mathrm{~W} / \mathrm{kg}$ (at 1 AU, beginning-of-life, operating temperature, $\sim 60 \mathrm{deg} C$ ) for flexible blanket arrays ${ }^{1}$. Flexible blanket arrays include, for example, the Ultraflex, manufactured by Northrup Grumman Innovation Systems, and Roll-Out 
Solar Array (ROSA), manufactured by Deployable Space Systems (DSS). These technologies are applicable to both Earth-orbiting and planetary science missions. It is estimated that many nextgeneration planetary science and astrobiology mission concepts will require solar cell efficiencies $\sim 38 \%$ and specific power $>200 \mathrm{~W} / \mathrm{kg}$ to fully meet their objectives ${ }^{1}$. The needs of planetary science and astrobiology missions and the technologies to address them are discussed below.

\section{Needs and Technologies for Outer Planet Missions}

Missions to the outer planets pose three key challenges for solar arrays. These include 1) low solar irradiance, which reduces the solar energy available, especially beyond Jupiter; 2) low temperatures, particularly during eclipse and offpointing, where temperatures as low as 33 deg $\mathrm{K}$ can jeopardize hardware and 3) high radiation environments for missions to the Jovian system. Existing missions, such as Juno ${ }^{3}$, have successfully demonstrated solar array technology at Jupiter (5.0 to $5.5 \mathrm{AU})$. The Juno spacecraft is shown in Figure 1. The upcoming Europa Clipper mission is establishing the capability to operate solar arrays at

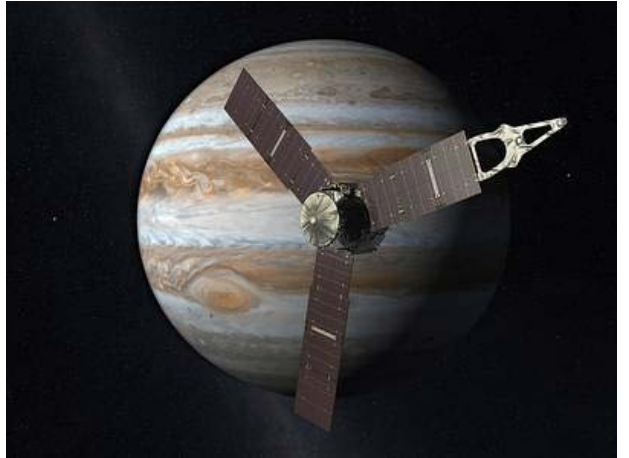

Figure 1. Juno Spacecraft. The Juno spacecraft is presently in Jovian orbit. Illustration is from www. nasa.gov/missionpages/juno/images/index.html. Jupiter in more severe radiation and thermal environments than $\mathrm{Juno}^{4}$.

Technology is currently under development to enable solar powered missions beyond Jupiter. Solar cells have been developed that are optimized for Saturn $(9.0 \text { to } 10 \mathrm{AU})^{5}$. Array-level technology incorporating these cells is under development as well. Saturn-optimized arrays would use lightweight structure and deployment technology, such as the flexible blankets and deployment mechanisms shown in Figure 2. NASA's Space Technology Mission Directorate recently completed a multiyear effort to combine LILT optimized solar cells, lightweight structures and reflective solar concentrators to improve performance for deep space missions ${ }^{6}$.

For Saturn-optimized arrays, specific power at Saturn is expected to reach $3 \mathrm{~W} / \mathrm{kg}$, which would make solar arrays an attractive alternative to radioisotope thermoelectric generators (RTGs) ${ }^{7}$. Continued development of this technology could enable future missions to Saturn and support the goals of the concept study, "Flagship Concepts for Astrobiology at Enceladus"».
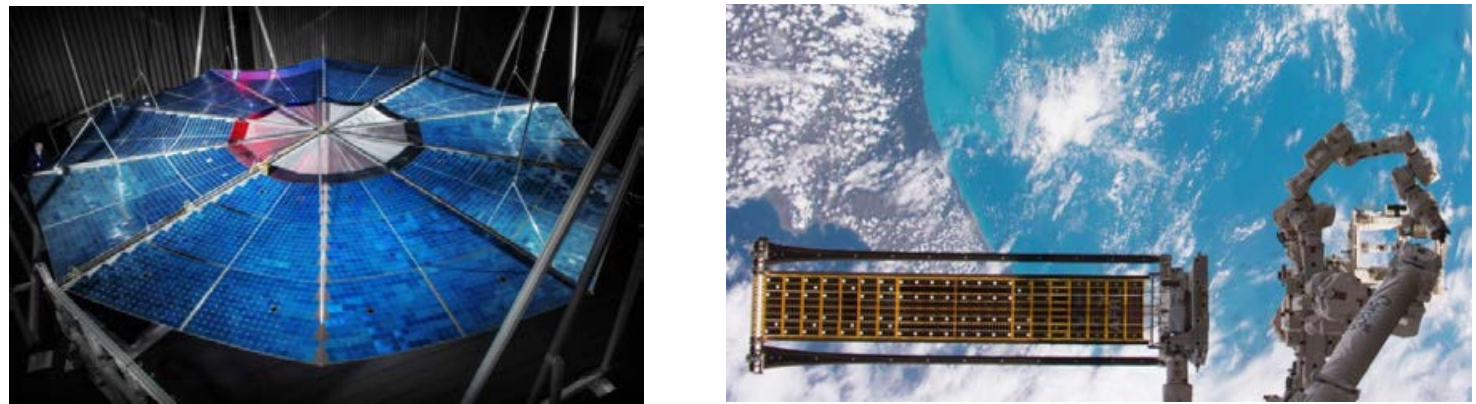

Figure 2. Flexible Blanket Solar Arrays. At left, a 10-m diameter MegaFlex demonstration unit was deployed in ground test ${ }^{9}$. The Megaflex is a larger version of the Ultraflex, which has been used on Mars landers and the Cygnus Commercial Resupply Vehicle. At right is a photograph of the Roll-Out Solar Array (ROSA) demonstrated on the International Space Station ${ }^{10}$. 
Initial work has begun on assessing whether solar arrays could be used for some mission concepts to Uranus. Using the technology under development for Saturn, it is estimated that a 100 $\mathrm{W}$ array could be built with a total area of $111 \mathrm{~m}^{2}$, approximately $10 \%$ larger than the Europa Clipper array. Specific power would only be $\sim 0.7 \mathrm{~W} / \mathrm{kg}$ and, therefore mass would be greater than an RTG. However, the cost of this approach may be substantially less than an RTG and, therefore, may enable a more economical mission to Uranus.

\section{Needs and Technologies for Inner Planet Missions}

Missions to the inner planets and the sun enjoy the benefits of abundant sunlight, but suffer the effects of extremely high temperatures. Solar arrays have been demonstrated, for example on the Parker Solar Probe, at 0.046 AU from the sun, using a pumped fluid loop for active thermal control $^{11}$. An illustration of the Parker Solar Probe is shown in Figure 3.

The Bepi Colombo mission, a pair of Mercury orbiters built by ESA and JAXA, is presently en route to Mercury and uses passive thermal control to manage solar array temperature $^{12}$. Specifically, second surface mirrors on the solar array and off-pointing are used to reduce the heat load at distances as low as $\sim 0.3 \mathrm{AU}$. Both passive and active thermal control can be applied to design solar arrays for a Mercury lander, although these are probably only compatible with a lander at high latitude.

Missions to the Venus atmosphere and surface present unique challenges, contending with highly corrosive gases, high pressure (93 bar at the surface), high temperatures (465 deg $\mathrm{C}$ at the surface), low solar irradiance ( $\sim 0.065$ suns at the surface) and a red-shifted spectrum"13. A "Venus Flagship Mission Concept" is under study as input to the decadal survey ${ }^{8}$.

The dependence of temperature, pressure and solar irradiance on altitude on Venus is shown Figure 4. Due to the wide range of conditions, no one solution will satisfy all possible missions. LIHT conditions will dominate at or near the surface while aerobots operating in the clouds will benefit from order-of-magnitude higher irradiance and significantly lower temperatures. Solar power on the surface of Venus and in the lower atmosphere had long been thought unfeasible, but research has progressed in this area. Key issues include

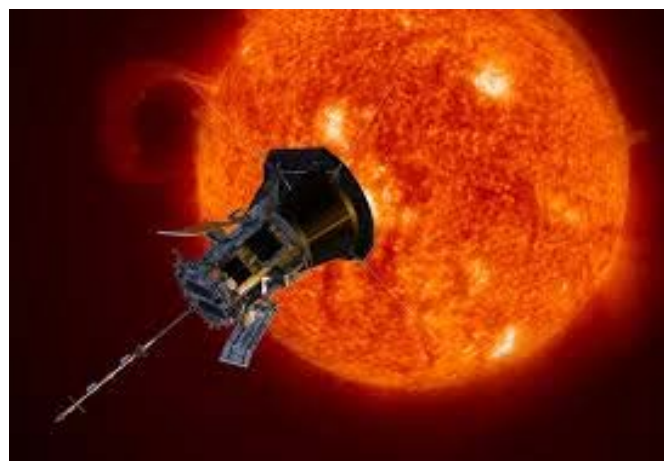

Figure 3. Parker Solar Probe. The Parker Solar Probe is en route to $0.046 \mathrm{AU}^{12}$. Illustration is from wikipedia.org/ wiki/Parker Solar Probe ${ }^{13}$.

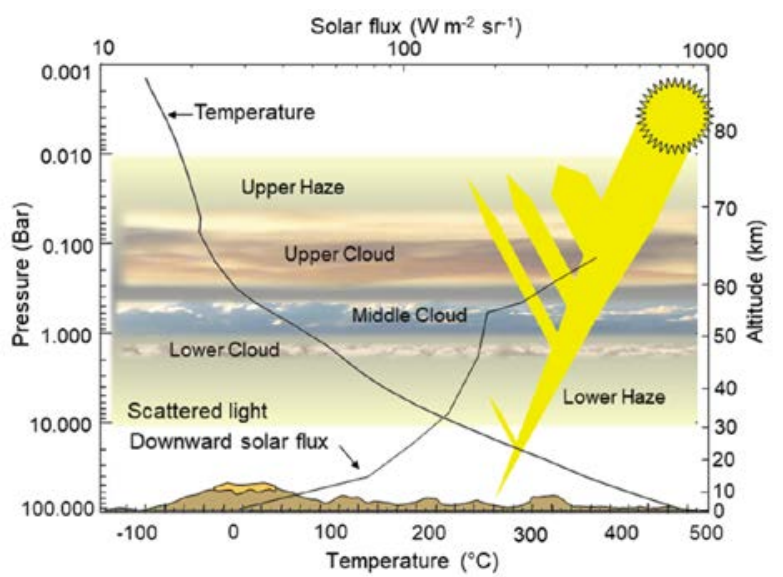

Figure 4. Venus Environment. Temperature and pressure increase dramatically and solar irradiance diminishes at lower altitudes ${ }^{14}$. 
degradation of solar cells due to diffusion of materials at high temperature, corrosion of cells and solar array materials and reduced efficiency at high temperature. A solar cell surviving at surface temperatures was demonstrated as part of the NASA HOTTech program ${ }^{14}$. Development of arraylevel technology capable of surviving the Venus surface environment is underway at JPL.

Development of the ability to control solar cell temperature coefficients may also improve available solar array power under LIHT conditions. Due to fundamental semiconductor properties, solar cell efficiency typically decreases as temperature increases. However, this effect may be mitigated with careful design of the solar cell device.

\section{Needs and Technologies for Mars Missions}

Missions to Mars include orbital, surface and aerial missions. Surface and aerial missions are the most challenging and are constrained by dust accumulation on solar arrays, variations in optical depth through the atmosphere and a red-shifted spectrum. Two Mars landers, Phoenix and InSight have used Ultraflex blanket arrays, shown in Figure 5, to maximize specific power. The solarpowered rovers, Sojourner, Spirit and Opportunity, have used rigid panels, also shown in Figure 5. Solar array area is highly constrained for rovers and, hence, high power conversion efficiency in the Martian environment is a key technology need for these missions. Dust mitigation is also valuable for long-term operation and is expected to benefit from the development of this technology for lunar surface missions, discussed below.
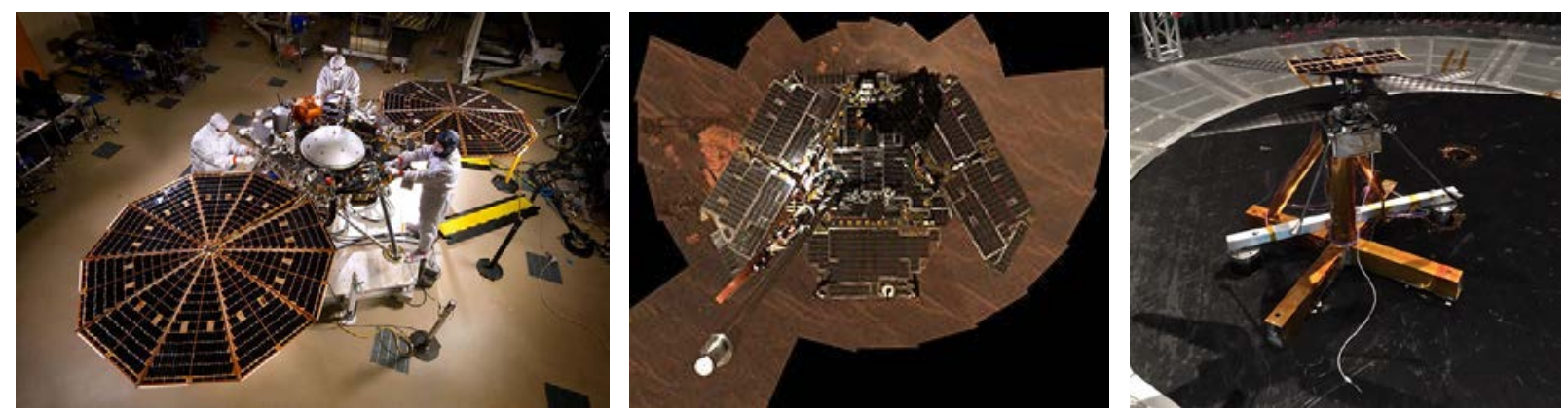

Figure 5. Mars Solar Arrays. At left is the InSight solar array, deployed for ground test (from wikipedia.org/wiki/ InSight). At center is a photograph of the Opportunity rover, taken on Mars (from wikipedia.org/wiki/Timelineof_Opportunity). At right, the Mars Helicopter is shown during test under simulated Martian conditions.

The Mars Helicopter, also shown in Figure 5, is a demonstration experiment scheduled for launch aboard the Mars 2020 spacecraft and planned for demonstration in 2021. The Mars Helicopter uses high efficiency IMM solar cells ${ }^{2}$ and a lightweight rigid panel to maximize specific power while surviving the launch and landing ${ }^{15}$. The solar array does not provide sufficient power for the helicopter to fly, so power during flight is provided by a rechargeable battery; the solar array is used to recharge the battery while the vehicle is on the surface. Future aerial mission concepts are expected to demand greater power, enabling a more capable payload and greater operating range. The key technology need for aerial systems is high specific power, since the solar array mass impacts the science payload capability and operating range.

\section{Needs and Technologies for Small Bodies}

Potential missions to small bodies span a variety of scenarios, such as landers on the asteroid Ceres, sample return from comets and exploration of the Trojan asteroids. Missions to distant 
bodies, such as the Trojans or Centaurs, share much in common with missions to outer planets. Solar arrays for these missions must operate under LILT conditions. In addition, large arrays are needed for such missions incorporating SEP. Hence, development of large, lightweight, LILToptimized arrays are needed for solar power to be an option for these applications.

Another capability is needed for solar-powered landers on asteroids in the special case where it is important for the lander to visit multiple locations on the asteroid. In this case, the solar array could deploy after landing, but then be retracted, so that the spacecraft can ascend and land again in a new location. Retraction can also enable a lander to perform sample return. Retractable solar arrays are possible in principle. In fact, the ROSA demonstrated on the International Space Station (ISS) was designed to be retractable. Further development and demonstration of this capability would enable a new type of mission for landers on small bodies. In particular, this would support the concept study, "Assessing Dwarf Planet Ceres Past and Present Habitability Potential”8.

\section{Needs and Technologies for Lunar Surface Missions}

Solar arrays have been on the lunar surface since the first Surveyor spacecraft landed in June 1966. The first lunar solar array provided $85 \mathrm{~W}$. Future lunar arrays will be needed for both rovers and central power stations. Power levels for stations can be expected to exceed $100 \mathrm{~kW}$, hence requiring much larger arrays for solar power solutions.

Lunar surface missions present two unique challenges. First, for high power systems, the solar array must deploy in the gravitational field on the lunar surface. The gravitational field is smaller than Mars'; however, the size of the array would be larger than previous Martian arrays. Second, the solar array will be vulnerable to dust accumulation and severe performance loss if long-term operation in the presence of human or robotic activity is required.

Development of large, deployable, high power arrays for the lunar surface can adapt either the rigid panel deployment systems or flexible blanket systems used on existing spacecraft. For example, the ROSA and Megaflex can be adapted to the lunar surface. NASA's Small Business Innovative Research (SBIR) program has recently sought proposals from industry to develop lunar polar surface arrays that deploy vertically from the surface, due to low sun angles at these locations.

Several research projects are underway to address the issue of dust accumulation. Approaches have included mechanical dust removal ${ }^{16}$, electrostatic methods ${ }^{17}$, plasmas $^{18}$ and electron beams $^{18,19}$. Development of this capability would enable a robust, reliable power source for longterm operations of both rovers and central power stations. In particular, it would support the goals

of two concept studies, "Intrepid Planetary Mission Concept (Long-Life Lunar Rover)"» and "Developing the Lunar Geophysical Network Mission"8.

\section{Developing Technologies Supporting a Broad Range of Missions}

While solar array technologies for planetary science and astrobiology missions have unique needs, as outlined above, this community benefits from ongoing progress in the broader photovoltaic (PV) community. For example, a monolithic, series-connected, six-junction inverted III-V metamorphic structure recently achieved $47.1 \%$ efficiency under the direct terrestrial solar spectrum at 143 Suns concentration, with a variation of the structure tuned to the global spectrum reaching a 1-Sun efficiency of $39.2 \%^{20}$. Significant effort is also being focused on reducing the 
growth costs of III-V PV materials through both dynamic hydride vapor phase epitaxy ${ }^{21}$ and ongoing efforts to decrease the cost of metal-organic chemical vapor deposition ${ }^{22}$. In addition, worldwide research on perovskite materials that can be deposited on lightweight, low cost substrates has resulted in rapidly increasing efficiencies for both single junction and multijunction structures. This technology offers the possibility of large, ultra-lightweight and lower cost space arrays in the future ${ }^{23}$, even with efficiencies that are generally lower than SoP solar cells.

Research and development of new array structures and deployment mechanisms may also be applicable to future science missions. Small flexible arrays, such as DSS's Aladdin, are being developed for small spacecraft ${ }^{24}$. Extremely large $(60 \mathrm{~m} \mathrm{x} 60 \mathrm{~m})$ flexible arrays intended for solar power satellites are being developed at Caltech ${ }^{25}$. These arrays may eventually be used to power missions to the outer planets. These represent just a few immediate examples of efforts that may impact space applications in the future.

\section{Summary and Recommendations}

Technology needs for solar arrays on next-generation planetary science and astrobiology missions include both common needs with Earth-orbiting systems and unique needs resulting from the special environments in which these missions operate. Common needs include higher efficiency solar cells, higher specific power arrays and larger arrays for higher power missions. Unique needs include survival and operation in extreme environments, such as LILT, LIHT, high radiation, extreme survival temperatures and dust accumulation. There are also some unique missions such as asteroid landers that could require retractable arrays.

Based on these observations, the following recommendations are intended to maximize NASA's investment in space solar array technology for planetary science and astrobiology:

1) NASA should make targeted investments in solar array technologies needed to meet the needs of its unique mission environments. Key areas include the following:

2) Solar arrays for the outer planets and moons, including Saturn and beyond, targeting high power ( $>100 \mathrm{~kW}$ at $1 \mathrm{AU})$ and low mass $(200-250 \mathrm{~W} / \mathrm{kg})$

3) Solar arrays for landers on moons and small bodies, including retractable mechanisms

4) Solar arrays for the Venus atmosphere and surface, tolerant over a range of conditions, including low irradiance, high temperature, high pressure and corrosive environments

5) Dust mitigation on lunar and Martian surfaces to enable long-term, reliable operation

6) Infrastructure for testing and qualification of solar array technology for unique environments, to enable rapid insertion of new technologies for unique mission needs

7) Partnerships with commercial space industry, DoD and other organizations to leverage ongoing developments in the areas of common needs. Specifically, leverage investments in high efficiency ( 38\% at AM0) solar cells enabling high power and low mass arrays.

\section{References}

1“Solar Power Technologies for Future Planetary Science Missions”, Jet Propulsion Laboratory document \#D-101316, December 2017

${ }^{2}$ solaerotech.com/wp-content/uploads/2018/04/IMM-alpha-Preliminary-Datasheet-April-2018

Research Laboratory, ESPC 2016, E3S Web of Conferences 16, 01001 (2017)

${ }^{3}$ www.nasa.gov/mission_pages/juno/spacecraft/index.html 
${ }^{4}$ Ulloa-Severino et al, "Power Subsystem Approach for the Europa Mission”, European Space Power Conference, 2016, E3S Web of Conferences 16, 13004 (2017)

${ }^{5}$ Andreea Boca et al, "Solar Power for Deep-Space Applications: State of Art and Development”, AIAA Propulsion Energy Forum 2019, AIAA 2019-4236

${ }^{6}$ Ed Gaddy et al, "Design of Solar Arrays for Deep Space Application”, 2018 Conference on Advanced Power Systems for Deep Space Application, Pasadena, CA, May 2018

${ }^{7}$ Terry J. Hendricks et al, "Solar Power System and Radioisotope Thermoelectric Generation Technologies at Jupiter-Saturn-Uranus Environments: New Insights and Paradigms”, 70th International Astronautical Congress, Washington, DC, IAC-19.C3.3.1.x54526

${ }^{8}$ Planetary Mission Concept Studies Workshop, Lunar \& Planetary Institute, Houston, TX, May 2020: https://www.hou.usra.edu/meetings/pmcs2020/format/

${ }^{9}$ www.orbitalatk.com/space-systems/space-components/solar-arrays/docs/FS008_15_OA_7463 \%20MegaFlex\%20Solar\%20Array.pdf

${ }^{10} \mathrm{https} / /$ technology.grc.nasa.gov/documents/Final_ISS_ROSA_Spaceflight_Information\%20 Release.pdf

${ }^{11}$ parkersolarprobe.jhuapl.edu/Spacecraft/index.php\#introduction

${ }^{12}$ sci.esa.int/web/bepicolombo/-/48872-spacecraft

${ }^{13}$ Hunten, D.M., Colin, L., Donahue, T. M., Moroz, V. I., Venus. 1983.

${ }^{14}$ J. Grandidier, A. P. Kirk, P. Jahelka, M. A. Stevens, P. K. Gogna, D. Crisp, et al., "Photovoltaic operation in the lower atmosphere and at the surface of Venus", Progress in Photovoltaics: Research and Applications, 28, 6 (2020)

${ }^{15}$ mars.nasa.gov/files/mars2020/Mars2020_Helicopter_Fact_Sheet.pdf

16، “Solar Array Dust Removal System (SADRS) for Long Life Mars Surface Missions Phase II Final Report”, ATK Space, JPL Contract No. 1264237, 2005

${ }^{17}$ C.A. Calle et al, "Dust Particle Removal by Electrostatic and Dielectrophoretic Forces with Applications to NASA Exploration Missions”, Proc. ESA Annual Meeting on Electrostatics (2008)

${ }^{18}$ T.M. Flanagan and J. Goree, "Dust Release from Surfaces Exposed to Plasma”, Physics of Plasmas 13, 123504 (2006)

${ }^{19}$ B. Farr, X. Wang, J. Goree, I. Hahn, U. Israelsson and M. Horányi (2020), "Dust mitigation technology for lunar exploration utilizing electron beam”, (submitted)

${ }^{20}$ John F. Geisz et. al., "Six-junction III-V solar cells with $47.1 \%$ conversion efficiency under 143 Suns concentration”, Nature Energy 5, 326 (2020)

${ }^{21}$ John Simon et. al., "III-V-Based Optoelectronics with Low-Cost Dynamic Hydride Vapor Phase Epitaxy”, Crystals 9, 3 (2019)

${ }^{22} \mathrm{R}$. Langa et. al., "MOVPE growth of GaAs with growth rates up to $280 \mu \mathrm{m} / \mathrm{h}$ ”, Journal of Crystal Growth 537, 125601 (2020)

${ }^{23}$ Dong-Nyuk Jeong, June-Mo Yang and Nam-Gyu Park, "Roadmap on halide perovskite and related devices”, Nanotechnology 21, 152001 (2020)

${ }^{24}$ J. Merrill et al, "Advanced Photovoltaic Power System Development at the U.S. Air Force Research Laboratory”, E3S Web of Conferences 16, 01001 (2017)

${ }^{25}$ Manan Arya, Nicolas Lee and Sergio Pellegrino, "Ultralight Structures for Space Solar Power Satellites”, $3^{\text {rd }}$ AIAA Spacecraft Structures Conference, January 2016 